\title{
Clinical characteristics of zinc phosphide poisoning in Thailand
}

\author{
Satariya Trakulsrichai ${ }^{1,2}$ \\ Natcha Kosanyawat' \\ Pongsakorn Atiksawedparit ${ }^{1}$ \\ Charuwan Sriapha ${ }^{2}$ \\ Achara Tongpoo ${ }^{2}$ \\ Umaporn Udomsubpayakul ${ }^{3}$ \\ Panee Rittilert ${ }^{2}$ \\ Winai Wananukul ${ }^{2}$ \\ 'Department of Emergency Medicine, \\ ${ }^{2}$ Ramathibodi Poison Center, ${ }^{3}$ Section \\ for Clinical Epidemiology and \\ Biostatistics, Research Center, Faculty \\ of Medicine, Ramathibodi Hospital, \\ Mahidol University, Bangkok, Thailand
}

Correspondence: Satariya Trakulsrichai Department of Emergency Medicine, Faculty of Medicine, Ramathibodi Hospital, Mahidol University, Rama VI Road, Bangkok 10400, Thailand

$\mathrm{Tel}+66220 \mathrm{I} \mid 484$

Fax +66 220I 2404

Email satariya.tra@mahidol.ac.th

\author{
This article was published in the following Dove Press journal: \\ Therapeutics and Clinical Risk Management \\ 14 March 2017 \\ Number of times this article has been viewed
}

Objective: The objectives of this study were to describe the clinical characteristics and outcomes of poisoning by zinc phosphide, a common rodenticide in Thailand, and to evaluate whether these outcomes can be prognosticated by the clinical presentation.

Materials and methods: A 3-year retrospective cohort study was performed using data from the Ramathibodi Poison Center Toxic Exposure Surveillance System.

Results: In total, 455 poisonings were identified. Most were males $(60.5 \%)$ and from the central region of Thailand $(71.0 \%)$. The mean age was $39.91 \pm 19.15$ years. The most common route of exposure was oral (99.3\%). Most patients showed normal vital signs, oxygen saturation, and consciousness at the first presentation. The three most common clinical presentations were gastrointestinal (GI; 68.8\%), cardiovascular (22.0\%), and respiratory (13.8\%) signs and symptoms. Most patients had normal blood chemistry laboratory results and chest X-ray findings at presentation. The median hospital stay was 2 days, and the mortality rate was $7 \%$. Approximately $70 \%$ of patients underwent GI decontamination, including gastric lavage and a single dose of activated charcoal. In all, 31 patients were intubated and required ventilator support. Inotropic drugs were given to $4.2 \%$ of patients. Four moribund patients also received hyperinsulinemia-euglycemia therapy and intravenous hydrocortisone; however, all died. Patients who survived and died showed significant differences in age, duration from taking zinc phosphide to hospital presentation, abnormal vital signs at presentation (tachycardia, low blood pressure, and tachypnea), acidosis, hypernatremia, hyperkalemia, in-hospital acute kidney injury, in-hospital hypoglycemia, endotracheal tube intubation, and inotropic requirement during hospitalization $(P<0.05)$.

Conclusion: Zinc phosphide poisoning causes fatalities. Most patients have mild symptoms, and GI symptoms are the most common. Patients who present with abnormal vital signs or electrolytes might have more severe poisoning and should be closely monitored and aggressively treated. All patients should be observed in the hospital for 2 days and followed up for cardiovascular and respiratory symptoms, electrolyte balances, kidney function, and blood glucose.

Keywords: zinc phosphide, poisoning, clinical characteristic, outcome

\section{Introduction}

Aluminum phosphide and zinc phosphide are effective fumigants and rodenticides widely used in many countries, especially in developing countries. Upon ingestion, phosphides come into contact with fluids in the gut and are converted to phosphine gas, which is then absorbed into the bloodstream. Phosphine is a highly toxic gas in humans and exerts its effects by many proposed mechanisms, including inhibition of cytochrome $\mathrm{C}$ oxidase and oxidative respiration. ${ }^{1,2}$ Phosphine mainly affects the cardiovascular, respiratory, gastrointestinal (GI), hepatobiliary, and hematologic systems and causes electrolyte and metabolic abnormalities. ${ }^{1,2}$ The severe clinical symptoms 
in patients with phosphine poisoning include circulatory collapse, hypotension, pulmonary edema, congestive heart failure, cardiac arrhythmia, and acute renal failure. ${ }^{1,3,4}$ No specific antidote has been identified; therefore, the main treatment is supportive care. Although some efforts have been made to establish more effective interventions and medications for management, the mortality rate remains high, especially for aluminum phosphide poisoning. Although aluminum phosphides and zinc phosphides generate phosphine gas in the human body, some clinical features and the mortality rates differ between these two metal phosphides. ${ }^{5,6}$

Zinc phosphide is a gray crystalline compound or gray black powder. ${ }^{1,2}$ In Thailand, zinc phosphide $\left(\mathrm{Zn}_{3} \mathrm{P}_{2}\right)$ is a commonly used household rodenticide because of its low cost and high availability. Clinical studies and reports of zinc phosphide poisoning in human beings are limited. ${ }^{3,7-11}$ Therefore, the present study was performed to ascertain the clinical characteristics, outcomes, and factors associated with death in cases of zinc phosphide poisoning in Thailand.

\section{Materials and methods Study design}

We performed a 3-year retrospective cohort study (July 2013June 2015) using data from the Ramathibodi Poison Center Toxic Exposure Surveillance System. The primary outcome was the clinical characteristics and outcome of all zinc phosphide poisoning cases. The secondary outcome was the factors associated with mortality.

This study was approved by the Institutional Ethics Committee Board of Ramathibodi Hospital, Faculty of Medicine, Mahidol University, and patient consent was deemed not necessary due to the retrospective nature of this study.

\section{Study setting and population}

The study setting was a poison center of a tertiary teaching hospital that was mainly consulted by medical personnel ( 15,000-20,000 consultations/year).

All patients with zinc phosphide exposure for whom consultation was performed at Ramathibodi Poison Center were included in the study. The diagnosis of zinc phosphide poisoning was based on clinical data, including a history of rodenticide ingestion with information on the brand name or characteristics of the rodenticide, and/or the patients brought the rodenticide containers with them to the hospital. The exclusion criterion was co-ingestion of other drugs, herbs, or pesticides.

\section{Study protocol}

We collected data on all patients included in the study. The data included demographic data, medical history, clinical features, laboratory results, treatment modalities, follow-up details, final diagnosis, and outcome.

Hypotension was defined as a systolic blood pressure of $<90 \mathrm{mmHg}$. Shock was identified as hypotension with signs of poor tissue perfusion. Jaundice was defined as characteristic clinical findings on physical examination with a plasma bilirubin concentration of $>1.5 \mathrm{mg} / \mathrm{dL}$. Hypoglycemia was defined as a plasma glucose concentration of $<60 \mathrm{mg} / \mathrm{dL}$ or was diagnosed based on the data obtained during the follow-up call. Acute kidney injury was diagnosed based on the clinical history and laboratory data using the Acute Kidney Injury Network criteria. We assumed that almost all patients were healthy and had normal kidney function before poisoning.

We used PASW Statistics for Windows, Version 18 (SPSS Inc., Chicago, IL, USA) to analyze the data. Continuous data were presented as mean and standard deviation, and categorical data were presented as frequency and percentage. Comparisons between the groups were analyzed by Student's $t$-test if the data were normally distributed and by the Mann-Whitney $U$ test otherwise. Differences in categorical variables were evaluated by chi-square analysis and Fisher's exact test. A $P$-value of $<0.05$ was considered statistically significant.

\section{Results}

In total, 489 patients with zinc phosphide exposure were identified. When we excluded patients with co-ingestion, 455 poisoned patients were included in our study. The circumstances of exposure were intentional poisoning or a suicidal attempt (84.4\%), accidental exposure (15.4\%), and occupational exposure $(0.2 \%)$. The route of exposure was either oral $(99.3 \%)$ or inhalation $(0.7 \%)$.

A total of 34 patients $(7.5 \%)$ had a history of drinking alcohol while taking zinc phosphide. Most patients (59.8\%) could not remember the exact brand name of the rodenticide; however, they were able to describe the characteristics of the rodenticide, such as the color of the powder. The patients' clinical characteristics and demographic data are shown in Table 1. The clinical presentations and vital signs of the patients are summarized in Table 2. Most patients presented with nausea and vomiting. Additionally, most patients had normal laboratory test results at the first presentation. Three patients underwent measurement of the serum cortisol concentration while in a state of shock. The cortisol concentration was $27.8 \mu \mathrm{g} / \mathrm{dL}$ in one patient and $>60 \mu \mathrm{g} / \mathrm{dL}$ in the other two patients. Among 26 patients, the chest X-ray findings were normal in 19 patients (73.1\%) and abnormal in 7 patients. The chest X-ray abnormalities were pulmonary edema in five patients (19.2\%) and infiltration in two patients (7.7\%). 
Table I Clinical characteristics of patients with zinc phosphide poisoning

\begin{tabular}{ll}
\hline Characteristics & $\mathbf{n}(\%)$ \\
\hline Sex & \\
$\quad$ Male & $277(60.9)$ \\
$\quad$ Female & $178(39.1)$ \\
Age in years, median (min-max) & $39.91(1-90)$ \\
Region & \\
Central & $157(34.5)$ \\
Northeast & $109(24.0)$ \\
North & $96(21.1)$ \\
South & $53(11.6)$ \\
West & $26(5.7)$ \\
East & $14(3.1)$ \\
Duration from zinc phosphide exposure to hospital & $1(0.083-120)$ \\
visit in hours, median (min-max) & \\
Amount of exposure & \\
$<$ I package & $207(45.5)$ \\
$>$ I package & $161(35.4)$ \\
Unknown & $87(19.1)$ \\
\hline
\end{tabular}

Abbreviations: min, minimum; max, maximum.

In 83 patients, an electrocardiogram (ECG) was obtained at presentation. The abnormalities discovered among these 83 patients were sinus tachycardia (18.1\%), atrial fibrillation $(4.8 \%)$, sinus bradycardia $(1.2 \%)$, and right bundle branch block (1.2\%).

Table 2 Clinical presentations and vital signs at presentation

\begin{tabular}{ll}
\hline Clinical presentations & $\mathbf{n}(\%)$ \\
\hline Common presenting symptoms & \\
Nausea and vomiting & $227(49.9)$ \\
Dyspnea & $56(12.3)$ \\
Drowsiness & $42(9.2)$ \\
Abdominal pain & $41(9.0)$ \\
Respiratory problem & $6(1.3)$ \\
Palpitation & $3(0.7)$ \\
Body temperature $\left({ }^{\circ} \mathrm{C}\right.$, peripheral; data & \\
available in 303 patients) & \\
$<37.5$ & $288(95)$ \\
$>37.5$ & $15(5.0)$ \\
Pulse rate (beats/minute; data & \\
available in 422 patients) & \\
$<60$ & $6(1.4)$ \\
$60-100$ & $356(84.4)$ \\
$>100$ & $60(14.2)$ \\
Respiratory rate (breaths/minute) & \\
$<20$ & $320(84.0)$ \\
$>20$ & $61(16.0)$ \\
Blood pressure (mmHg) & \\
Low & $23(5.5)$ \\
Normal & $349(78.9)$ \\
High & $74(17.6)$ \\
Oxygen saturation from pulse oximeter & \\
Normal & $74(97.4)$ \\
Low & $2(2.6)$ \\
Glasgow Coma Scale score & \\
$<9$ & $9(2.0)$ \\
$9-12$ & $11(2.4)$ \\
I3-15 & $430(95.6)$ \\
\hline &
\end{tabular}

Treatment modalities included intravenous fluids (85.3\%), gastric lavage (74.7\%), activated charcoal (73.2\%), and oxygen therapy $(9.7 \%)$. Endotracheal intubation was performed in $31(6.8 \%)$ patients, inotropic drug infusion in $19(4.2 \%)$ patients, hydrocortisone administration in $4(0.9 \%)$ patients, and hyperinsulinemia-euglycemia therapy in $4(0.9 \%)$ patients. Almost all patients $(97.1 \%)$ were admitted to the hospital, usually to the general ward (94.3\% of all admitted patients). The median length of hospital stay was 2 days (range, 1-9 days). The mortality rate in our study was 7\% (32 patients). Of the 32 patients who died, 2 had accidentally ingested zinc phosphide and 30 had attempted to commit suicide (intentional ingestion). Most of the patients who died (75\%) developed systemic symptoms such as cardiovascular or respiratory symptoms within 24 hours after ingestion, while the others (25\%) developed systemic symptoms $24-48$ hours after ingestion. A total of 17 patients (53.1\% of deaths) developed systemic symptoms and died within 24 hours after ingestion. Ten (31.3\%) and four patients (12.5\%) died 24-48 and 48-72 hours after ingestion, respectively. One patient developed systemic symptoms on the first day of ingestion, but died the fifth day after ingestion; however, his death was not caused by the zinc phosphide ingestion. Among the patients who died, those who developed systemic symptoms died within the first 48 hours after development of these symptoms. In all, 15 patients who died underwent an ECG recording while they were in cardiac arrest. The ECGs of five, nine, and one patient showed ventricular tachycardia or ventricular fibrillation, pulseless electrical activity, and asystole, respectively.

We performed a subgroup analysis of the factors during admission that were associated with in-hospital mortality between the patients who died and survived. All 442 patients who were admitted to the hospital were analyzed. The clinical characteristics and laboratory findings are compared between the patients who survived and died in Table 3 . The factors that were significantly different between the patients who survived and died were age, duration from zinc phosphide exposure to the hospital visit, abnormal vital signs at presentation (tachycardia, low blood pressure, or shock and tachypnea), acidosis, hypernatremia, hyperkalemia, in-hospital acute kidney injury, in-hospital hypoglycemia, endotracheal tube intubation, and inotropic requirement during hospitalization. No significant differences were found in sex, alcohol co-ingestion, temperature at presentation, oxygen saturation at presentation, Glasgow coma scale score at presentation $(<8,9-12$, or $>13)$, total bilirubin concentration, gastric lavage, or activated charcoal administration. The amount of zinc phosphide ingested could not be precisely 
Table 3 Statistically significant differences in clinical characteristics and laboratory findings between patients who survived and died

\begin{tabular}{|c|c|c|c|}
\hline Clinical characteristics and laboratory findings, number of patients & Patients who died & Patients who survived & $P$-value \\
\hline Age, mean $\pm S D, 442$ patients & $49.09 \pm 14.588$ & $39.43 \pm 19.412$ & 0.006 \\
\hline $\begin{array}{l}\text { Duration from zinc phosphide exposure to hospital visit in hours, median } \\
\text { (min-max), } 442 \text { patients }\end{array}$ & $3(0.33-24)$ & I $(0.083-120)$ & 0.001 \\
\hline Tachycardia at presentation ( $>100$ beats/minute), $\%$ of $4 \mathrm{II}$ patients & 33.3 & 13.1 & 0.015 \\
\hline Low systolic blood pressure at presentation ( $<90 \mathrm{mmHg}$ ), \% of 410 patients & 30.0 & 1.6 & $<0.001$ \\
\hline Low diastolic blood pressure at presentation $(<60 \mathrm{mmHg}), \%$ of 409 patients & 33.3 & 3.4 & $<0.001$ \\
\hline Tachypnea at presentation ( $>20$ breaths/minute), $\%$ of 370 patients & 38.5 & 14.5 & 0.004 \\
\hline Hypernatremia at presentation ( $>145 \mathrm{mmol} / \mathrm{L}), \%$ of 328 patients & 25.0 & 3.9 & 0.001 \\
\hline Hyperkalemia at presentation ( $>5.5 \mathrm{mmol} / \mathrm{L}), \%$ of 330 patients & 8.3 & 0.7 & 0.014 \\
\hline Acidosis (by electrolyte analysis) at presentation, \% of 324 patients & 60.9 & 8.0 & $<0.001$ \\
\hline Acute kidney injury, \% of 309 patients & 50.0 & 6.3 & $<0.001$ \\
\hline Hypoglycemia, \% of 92 patients & 40.0 & 3.7 & $<0.001$ \\
\hline Endotracheal tube intubation, $\%$ of 442 patients & 68.8 & 2.2 & $<0.001$ \\
\hline Inotropic agent administration, $\%$ of 442 patients & 94.7 & 5.3 & $<0.001$ \\
\hline
\end{tabular}

Abbreviations: SD, standard deviation; min, minimum; max, maximum.

estimated; therefore, we did not include this amount as a factor to be analyzed.

\section{Discussion}

Zinc phosphide is legal, inexpensive, and readily available in Thailand. According to our data, it is a commonly used rodenticide involved in $\sim 150$ cases of poisoning per year from every region of Thailand. It is also a common deliberate self-poisoning agent. The most common presentation in the present study was mild GI symptoms such as nausea and vomiting, and most patients had normal laboratory test results at the first presentation.

In one study, the authors stated that zinc phosphide rodenticide poisoning is a cause of acute liver failure in many Indian patients. ${ }^{12}$ Interestingly, none of the patients in the present study developed severe liver injury or acute liver failure during hospital admission. This differs from the findings of several other studies. ${ }^{12-14}$ This discrepancy might be partially explained by the differences in the zinc phosphide products among countries, for example, the composition or the substances added might be different.

Both hyperglycemia and hypoglycemia have been reported as clinical features of phosphide poisoning. . $^{1,8,11,15-18}$ Hypoglycemia may be persistent and severe. ${ }^{1,8}$ In the present study, hypoglycemia was the clinical feature of zinc phosphide poisoning, particularly in severe cases and in patients who died.

The chest X-ray and ECG at the first presentation were normal in most patients. However, some patients developed severe symptoms and signs later; therefore, close observation of patients with zinc phosphide poisoning is necessary. Most patients developed systemic symptoms within the first
24 hours; however, the others developed such symptoms in 24-48 hours. On the basis of these findings, we recommend that patients are observed in the hospital for 2 days.

The onset of symptoms in patients with aluminum phosphide poisoning is typically very rapid. The difference in the symptom onset times between these two metal phosphides might be partially explained by the fact that aluminum phosphide is unstable; this results in the rapid onset of systemic toxicity after ingestion. ${ }^{1,6}$ In contrast, zinc phosphide is a relatively stable compound. Another proposed explanation is that phosphine might take part in another reaction to form an intermediate compound. ${ }^{6}$ The present study confirmed this clinical feature of a delayed interval between ingestion of zinc phosphide and the appearance of systemic toxicity, even in patients who had already developed GI symptoms. In accordance with the ECG findings during cardiac arrest, the deaths in this study might have also been caused by various systemic abnormalities. However, an ECG recording during cardiac arrest was performed in only a small number of patients who died.

Most patients in the present study underwent GI decontamination, including gastric lavage and activated charcoal administration. This indicates that most of the medical institutions included these procedures in their poisoning management protocol. Although secondary exposure has been reported, ${ }^{1,19}$ our follow-up data indicate that no secondary exposure among the medical personnel was either recorded or consulted about after the gastric lavage procedure. However, the specialist in the poison information center who followed up the patients by telephone might not have specifically inquired about secondary exposure following this procedure. We cannot definitively conclude that no secondary contamination occurred, but 
exposure of the medical personnel to zinc phosphide was not obviously detected. Hydrocortisone and hyperinsulinemiaeuglycemia therapies were administered to severely poisoned patients who developed shock; however, these patients eventually died. When this finding was combined with the serum cortisol level, especially in the two patients with a serum cortisol concentration of $>60 \mu \mathrm{g} / \mathrm{dL}$ during shock, we considered that an adrenal crisis, including steroid therapy, might not be a main contributor in this poisoning. Although some studies ${ }^{20,21}$ have found that phosphide toxicity causes severe changes to the adrenal gland histopathology and affects adrenocortical involvement, these studies were performed in patients with aluminum phosphide poisoning. Further studies are required to clarify the effects on the adrenal system and benefits of treatment for zinc phosphide poisoning, particularly in critically ill patients.

The mortality rate in our study was 7\% (32 patients), which is lower than that of aluminum phosphide poisoning. Although some patients accidentally ingested the zinc phosphide, they died. Therefore, monitoring of all patients with zinc phosphide poisoning is necessary.

In one study of zinc phosphide poisoning, ${ }^{5}$ only 4 of 102 patients died. Conversely, in a smaller study, 5 of 20 patients died ( $25 \%$ mortality rate). ${ }^{3}$ Therefore, the mortality rate in our study, which included 455 patients, was low $(<10 \%)$. However, the other studies were performed in different countries that might have used different zinc phosphide products or different management protocols; this may have contributed to the differences in the mortality rates.

We found that older patients, those who visited the hospital late or received the initial treatment late, and those who had abnormal vital signs or electrolyte concentrations at presentation might represent high-risk patients who should be closely monitored or receive early supportive treatment. Their cardiovascular and respiratory symptoms should be observed. Additionally, their electrolyte concentrations, renal function, and blood glucose level should be monitored because abnormalities in these laboratory parameters are associated with mortality.

The factors associated with death in our study were consistent with the factors identified in other studies of phosphide poisoning. ${ }^{22,23}$ However, the low Glasgow Coma Scale score at presentation was not associated with mortality in our study; this differs from the findings in one other study. ${ }^{22}$ The authors of that study found that shock and an altered mental status were prognostic factors in patients with aluminum phosphide poisoning. ${ }^{22}$ This may be clarified by the possibility that the alcohol co-ingestion in some of our patients caused their depression of consciousness and might not have been associated with the systemic symptoms.

Our study had some limitations. First, it was a retrospective study and might have contained incomplete data. Second, the diagnosis mainly depended on the history that the patients reported to the medical personnel; therefore, not all histories may have been clear or completely accurate. Finally, no laboratory data could be used to definitively confirm the diagnosis of zinc phosphide poisoning and some blood chemistry results such as those of arterial blood gas analysis could not be obtained because of the limited resources of the laboratories in small hospitals.

\section{Conclusion}

Zinc phosphide poisoning still causes fatalities. Most patients have mild symptoms, and GI symptoms are the most common. The patients in our study presented with abnormal vital signs (especially, shock, tachypnea, and tachycardia) or abnormal electrolytes (especially acidosis); such patients should be closely monitored and aggressively treated. Patients should be observed in the hospital for 2 days, and their electrolyte concentrations, kidney function, and blood glucose levels should be followed up. Cardiovascular and respiratory problems should be observed because they are the cause of death in some patients.

\section{Acknowledgments}

The abstract of this article was presented at the 36th International Congress of the European Association of Poisons Centres and Clinical Toxicologists (EAPCCT) 24-27 May, 2016, Madrid, Spain, as a poster presentation with interim findings. The poster's abstract was published in "Poster Abstracts" in Clinical Toxicology: http://www.eapcct2016. com/images/site/EAPCCT 2016 congress abstracts.pdf.

\section{Disclosure}

The authors report no conflicts of interest in this work.

\section{References}

1. Proudfoot AT. Aluminium and zinc phosphide poisoning. Clin Toxicol (Phila). 2009;47(2):89-100.

2. Bumbrah GS, Krishan K, Kanchan T, Sharma M, Sodhi GS. Phosphide poisoning: a review of literature. Forensic Sci Int. 2012;214(1-3):1-6.

3. Chugh SN, Aggarwal HK, Mahajan SK. Zinc phosphide intoxication symptoms: analysis of 20 cases. Int J Clin Pharmacol Ther. 1998;36(7): 406-407.

4. Dogan E, Guzel A, Ciftci T, et al. Zinc phosphide poisoning. Case Rep Crit Care. 2014;2014:589712.

5. Hassanian-Moghaddam H, Shahnazi M, Zamani N, Rahimi M, Bahrami-Motlagh H, Amiri H. Plain abdominal radiography: a powerful tool to prognosticate outcome in patients with zinc phosphide poisoning. Clin Radiol. 2014;69(10):1062-1065. 
6. Marashi SM. What really happens after zinc phosphide ingestion? A debate against the current proposed mechanism of phosphine liberation in zinc phosphide poisoning. Eur Rev Med Pharmacol Sci. 2015; 19(22):4210-4211.

7. Kamal Ael A, Abd el-Hamid DM, Hatch DL, Ahmed Ael N. Epidemiological study of the claimed zinc phosphide intoxication in Kom Ombo district April 1996. J Egypt Public Health Assoc. 1999;74(1-2): 175-191.

8. Frangides CY, Pneumatikos IA. Persistent severe hypoglycemia in acute zinc phosphide poisoning. Intensive Care Med. 2002;28(2):223.

9. Sangle SA, Thomas A, Verma S, Wadia RS. Zinc phosphide poisoning. J Assoc Physicians India. 1987;35(8):591-594.

10. Rodenberg HD, Chang CC, Watson WA. Zinc phosphide ingestion: a case report and review. Vet Hum Toxicol. 1989;31(6):559-562.

11. Patial RK, Bansal SK, Kashyap S, Sharma AK, Sharma B. Hypoglycaemia following zinc phosphide poisoning. J Assoc Physicians India. 1990;38(4):306-307.

12. Saraf V, Pande S, Gopalakrishnan U, et al. Acute liver failure due to zinc phosphide containing rodenticide poisoning: clinical features and prognostic indicators of need for liver transplantation. Indian $J$ Gastroenterol. 2015;34(4):325-329.

13. Chittora MD, Meena SR, Gupta DK, Bhargava S. Acute hepatic failure in aluminium phosphide poisoning. J Assoc Physicians India. 1994; 42(11):924.

14. Bayazit AK, Noyan A, Anarat A. A child with hepatic and renal failure caused by aluminum phosphide. Nephron. 2000;86(4):517.

15. Mehrpour O, Alfred S, Shadnia S, et al. Hyperglycemia in acute aluminum phosphide poisoning as a potential prognostic factor. Hum Exp Toxicol. 2008;27(7):591-595.
16. Jain J, Jain VV, Gupta OP, Jaikishen A. Transient hyperglycemia in zinc phosphide poisoning. Indian J Endocrinol Metab. 2012;16(1): 145-146.

17. Jamshed N, Ekka M, Aggarwal P, Narayan S. Severe hypoglycemia as a presenting feature of aluminum phosphide poisoning. Ann Saudi Med. 2014;34(2):189.

18. Mehrpour O, Aghabiklooei A, Abdollahi M, Singh S. Severe hypoglycemia following acute aluminum phosphide (rice tablet) poisoning; a case report and review of the literature. Acta Med Iran. 2012;50(8): $568-571$.

19. Centers for Disease Control and Prevention (CDC). Occupational phosphine gas poisoning at veterinary hospitals from dogs that ingested zinc phosphide - Michigan, Iowa, and Washington, 2006-2011. MMWR Morb Mortal Wkly Rep. 2012;61(16):286-288.

20. Arora B, Punia RS, Kalra R, Chugh SN, Arora DR. Histopathological changes in aluminium phosphide poisoning. $J$ Indian Med Assoc. 1995;93(10):380-381.

21. Chugh SN, Ram S, Sharma A, Arora BB, Saini AS, Malhotra KC. Adrenocortical involvement in aluminium phosphide poisoning. Indian J Med Res. 1989;90:289-294.

22. Louriz M, Dendane T, Abidi K, Madani N, Abouqal R, Zeggwagh AA. Prognostic factors of acute aluminum phosphide poisoning. Indian $J$ Med Sci. 2009;63(6):227-234.

23. Mathai A, Bhanu MS. Acute aluminium phosphide poisoning: can we predict mortality? Indian J Anaesth. 2010;54(4):302-307.
Therapeutics and Clinical Risk Management

\section{Publish your work in this journal}

Therapeutics and Clinical Risk Management is an international, peerreviewed journal of clinical therapeutics and risk management, focusing on concise rapid reporting of clinical studies in all therapeutic areas outcomes, safety, and programs for the effective, safe, and sustained use of medicines. This journal is indexed on PubMed Central, CAS,

\section{Dovepress}

EMBase, Scopus and the Elsevier Bibliographic databases. The manuscript management system is completely online and includes a very quick and fair peer-review system, which is all easy to use. Visit http://www.dovepress.com/testimonials.php to read real quotes from published authors. 